\title{
Amonafide Dihydrochloride
}

National Cancer Institute

\section{Source}

National Cancer Institute. Amonafide Dihydrochloride. NCI Thesaurus. Code C999.

The dihydrochloride salt of amonafide, an imide derivative of naphthalic acid. Amonafide intercalates into DNA and inhibits topoisomerase II, resulting in protein-associated strand breaks and impaired DNA and RNA synthesis. 